\title{
Sensor Density for Full-View Problem in Heterogeneous Deployed Camera Sensor Networks
}

\author{
Zhimin Liu' ${ }^{1}$, Guiyan Jiang ${ }^{2 *}$ \\ ${ }^{1}$ School of Mathematics and Computational Science, Hunan First Normal University, Changsha, 410002,China \\ [e-mail: zhiminl@qq.com] \\ ${ }^{2}$ School of Economics, Wuhan University of Technology, Wuhan, 430070, China \\ [e-mail: jianggui_0028@qq.com] \\ *Corresponding author: Guiyan Jiang
}

Received May 12, 2021; revised July 19, 2021; accepted October 11, 2021;

published December 31, 2021

\begin{abstract}
In camera sensor networks (CSNs), in order to better identify the point, full-view problem requires capture any facing direction of target (point or intruder), and its coverage prediction and sensor density issues are more complicated. At present, a lot of research supposes that a large number of homogeneous camera sensors are randomly distributed in a bounded square monitoring region to obtain full-view rate which is close to 1 . In this paper, we deduce the sensor density prediction model in heterogeneous deployed CSNs with arbitrary full-view rate. Aiming to reduce the influence of boundary effect, we introduce the concepts of expanded monitoring region and maximum detection area. Besides, in order to verify the performance of the proposed sensor density model, we carried out different scenarios in simulation experiments to verify the theoretical results. The simulation results indicate that the proposed model can effectively predict the sensor density with arbitrary full-view rate.
\end{abstract}

Keywords: Camera sensor networks, full view, sensor density, sensor prediction 


\section{Introduction}

Currently, with the development of wireless Internet of things technology, the application of CSNs is more and more extensive, mainly used in medical monitoring, intelligent transportation, anti-terrorism detection, et al [1-3]. At the same time, there are many scholars engaged in such research fields of CSNs. Different from traditional wireless sensor networks (WSNs), the wireless sensor networks can not deal with complex task, while CSNs can handle complex tasks, such as real-time data processing, adaptive sensor direction adjustment, point recognition and tracking, et al [4]. When a effective facing direction of target (point or intruder) is captured by a camera sensor, CSNs can more accurately recognize and track point by using target recognition algorithm, so as to achieve better coverage monitoring. Due to the real-time mobility of point, it is very important for camera sensors to collaboratively work and capture any effective facing direction of point.

Coverage is one of the research highlights in CSNs. Ordinarily, the coverage are usually divided into three categories, mainly including area coverage, target coverage and barrier coverage. In these coverage issues, it is not necessary to consider the facing and moving directoin of intruders. However, in some CSN applications with high security requirements and real-time monitoring of intruder's face information. The CSN needs to real-time detect the intruder and captures the effective front image of intruder. Based on these requirements, Wang et, al. [5] presented the problem of full-view coverage and derived the condition of an intruder to be full-view covered for the first time. For any moving and face direction of the intruder, there is at least one sensor can monitor the intruder and capture the effective front image. In general, the intruder is called to be full-view covered, As illustrated in Fig. 1.

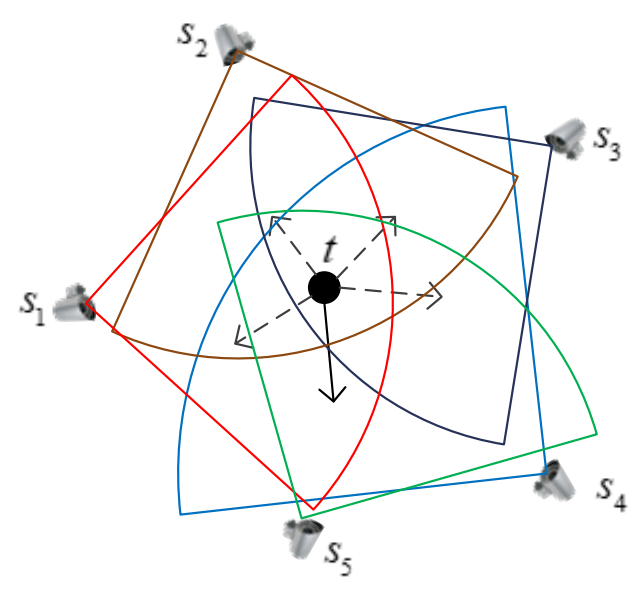

Fig. 1. Illustration of an intruder to be full-view covered

Due to most of the application scenarios of CSNs are unreachable, in general, each camera sensor in CSNs is stochastically deployed in the monitoring region. After the CSN is established, it is difficult to obtain the full-view rate of CSN. In order to guide engineers to design CSNs, it is very important to predict the sensor density of CSNs with arbitrary fullview rate. 
In this paper, we take the sensor density prediction problem of heterogeneous CSNs with arbitrary full-view rate into account. The existing literatures [5][7] studies the critical parameter issue of sensors with full-view probablity equal to 1 . However, these works can not be directly applied to solve the sensor density issue under an arbitrary full-view rate. As far as we know, there is no relevant research to study sensor density issue under an arbitrary fullview rate in heterogeneous CSNs. The main contributions of this paper include the following.

- We present the definitions of expanded monitoring region and maximum detected area.

- We derive a sensor density prediction model for heterogeneous CSNs with arbitrary full-view rate.

- We have put up the analysis of the performance of the model theoretical results and scenario simulation results.

The rest of this paper is arranged as follows. In section 2, the relevant models, scenario and definitions are introduced. Section 3 introduces the sensor density prediction model under an arbitrary full-view rate in detail. Section 4 introduces serveral simulation experiments. In section 5 , the paper is concluded.

\section{Related Work}

Recently, full-view coverage problem in CSNs has attracted wide attention of researchers. Literatures [5-6] presented the concept of full-view coverage in CSNs, and deduced the decsion conditon of full-view coverage. At the same time, the full-view prediction problem of uniform CSN based on equlateral triangle deployment is studied, but the boundary effect and the full-veiw prediction problem under arbitrary rate of heterogeneous CSNs were not considered. Hu et al. [7] studied the critical parameter prediction issue with full-view rate close to 1 in mobile CSN which is heterogeneous deployed. However, the research can not be directly applied to predict the sensor' scale in CSNs with arbitrary full-view rate. Authors [8] taken the asymptotic full-view problem in mobile CSN which is heterogeneous deployed. Besides, they unraveled the crucial conditions to achieve asymptotic full-view close to 1 . In literature [9], the concept and definition of local face-view barrier is proposed, and the prediction expression of local face-view barrier in CSN under deterministic deployment is derived. Wang et al. [10] analyzed the full-view barrier issue in homogeneous CSNs, and divided the target area into different subareas in which each subarea is covered by the same camera sensors. Meanwhile, they defined the problem as a shortest path problem and studied the deterministic deployment method to obtain full-view barrier with the minimum number of sensors.

Liu et al. [11] studied the full-view barrier coverage issue in mobile camera sensor networks, and divided the deployed region into some several connnected grids, then presented grid-based deployment algorithm to deploy camera sensors for each grid. At last, a weighted directed graph is constructed and a Dijkstra algorithm is proposed to obtain a shortest coverage barrier. Aiming to the enhancement problem of full-view barrier in homogeneous deployed CSNs, authors [12] presented a distributed algorithm to change the sensor's sensing direction so as to construct the cover-set. In literature [13], a novel distributed algorithm was presented to improve the fullv-view monitoring of quality in homogeneous deployed CSNs. In literature [15], the problem of full-view with minimum number of sensors in homogeneous deployed CSNs is studied. In addition, authors have proved that the issue can be simplified to target fullview problem, and presented two algorithms to establish the cover sets in which each cover set can get full-view rate close to 1. Literature [15] taken analysis of the maximum lifetime of full-view coverage problem in homogeneous deployed CSNs. Chen et al. [16] divided the 
direction space of camera sensors into a group of discrete directions, and proposed an algorithm ot select minimnum number of sensors with a sensing direction to obtain the fullview probality. Authors [17] studied area coverage prediction in heterogeneous CSNs based on boundary deployment, and conducted a series of simulations to verifty the accuracy of the proposed model. Khan et al. [18] presented an algorithm to improve the energy efficiency of instant message group chat messages.

\section{Problem statement and symbols}

In this section, the deployment scenario, the sensor's sensing model and the definition of full-view problem in heterogeneous deployed CSNs is introduced. Finally, the descriptions of symbols used in this paper are intruduced.

\subsection{Sensing model}

As illustrated in Fig. 2, the sensing model of a camera sensor is denoted as a 4-tuple $\langle s, r, \varphi, \stackrel{v}{v}\rangle$, where, $r$ denotes the sensing radius, $\varphi$ and $\stackrel{v}{v}$ represent the field-of-view (Fov) and the orientation of a camera sensors respectively. Without lossof generality, we use $s$ to denote the camera sensor and its position. Thse sensing sector determined by $\langle s, r, \varphi, v\rangle$ is called as sensing region, represented as $|s|$.

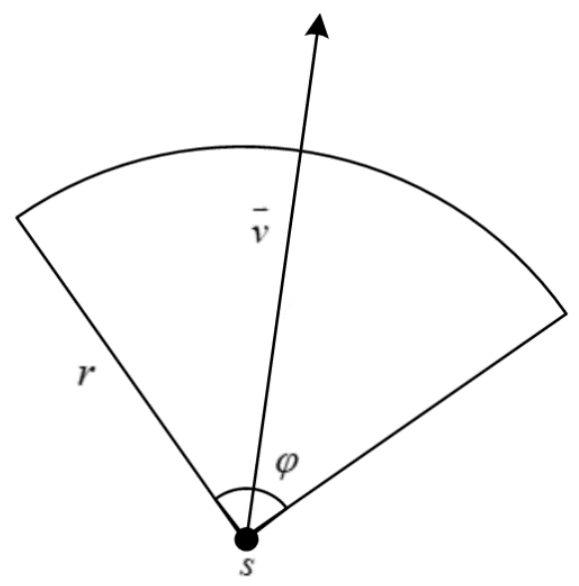

Fig. 2. Illustration of sensing radius

\subsection{Deployment model}

In this paper, we suppose that different groups of camera sensors which are stochastically arranged in a bounded square monitoring region denoted as $R$ with side length $L$ have different sensor density $\lambda$, sensing radius $r$, and field-of-view (Fov) angle $\varphi$. For simplicity, we consider such scenario that two groups of camera sensors denoted as $G_{1}$ and $G_{2}$ (also called Type I and Type II) with sensor density $\lambda_{1}$ and $\lambda_{2}$, sensing radius $r_{1}$ and $r_{2}$, Fov angle $\varphi_{1}$ and $\varphi_{2}$, are randomly scattered in region $R$. 
Due to a huge number of sensors are stochastically arranged in the monitoring region $R$, in this case, some sensors will fall on the boundary of the region monitoring region so as to result in the boundary effect. Aiming to reduce the influence of boundary effect on the monitoring of quality, the monitoring region is expanded as a new region, which is called expanded region with side length $L+r_{1}+r_{2}$, we denote the expanded region as $E R$. As shown in Fig. 3, all targets (points or intruders) are deployed in the monitoring region, while the camera sensors $\left(G_{1}\right.$ and $G_{2}$ ) are stochastically deployed in the expanded region.

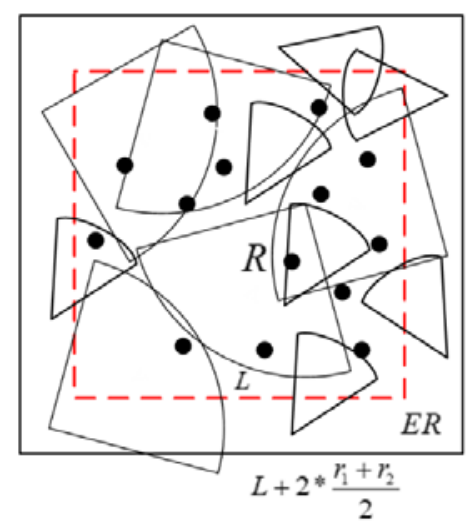

Fig. 3. Illustration of deployment model

\subsection{Definitions and theorem}

To better describe the full-view issue in CSNs, the relevant definitions and theorem are proposed.

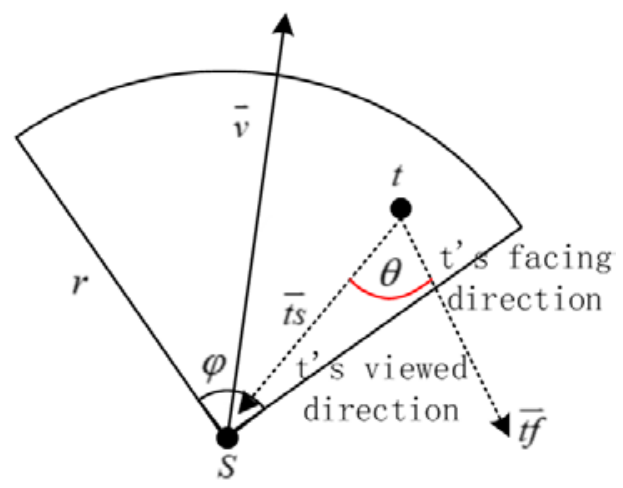

Fig. 4. Illustration of $\theta$-coverage

\section{1) $\theta$-Coverage}

As shown in Fig. 4, for a facing direction $\vec{t} f$ of the point $t$ in the monitoring region, it achieves $\theta$-Coverage if and only if it is covered by at least one camera sensor and the angle between $\overline{t f}$ and its viewed direction $t s$ is less than or equal to $2 \theta, \theta \in(0, \pi / 2)$, which is a 
preset constant called effective angle. If the following three conditions are met, we call $t \mathbf{u}$ is $\theta$-covered.

- $|s t| \leq r$. Here, st denotes the Euler distance between point $t$ and camera sensor $s$

- $\quad \alpha(\stackrel{\mathbf{u}}{s t}, \stackrel{r}{v}) \leq \varphi / 2$. Here, st denotes the anti-viewed direction of point $t ; \dot{v}$ represents the current sensing direction of sensor $s$.

- $\quad \alpha(t s, t f) \leq 2 \theta$. Here, $\stackrel{\mathbf{u}}{\mathbf{u}} \mathbf{u}$ and $\stackrel{\mathbf{u}}{t f}$ denote the viewed and facing direction of point $t$, respectively.

\section{2) Full-view Covered}

If any facing direction $t f$ of point $t$ is $\theta$-covered, we call the point $t$ is full-view covered.

\section{3) Detected Area}

For a point $t$, its detected region denoted as $d(t)$ is defined as a circular area with the sensing radius $r$ of sensor as the radius and the point $t$ as the center. Therefore, for different groups ( $G_{1}$ and $G_{2}$ ) of sensors, the detected area of the point $t$ is differeent, denoted as $d(t)_{1}$ and $d(t)_{2}$, respectively, as illustrated in Fig. 5.

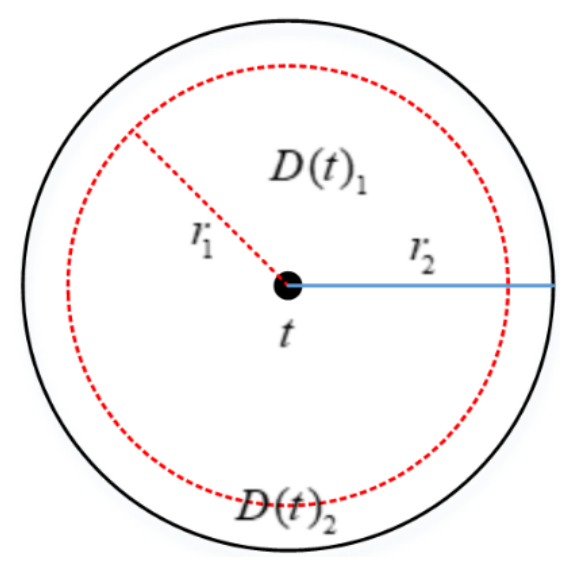

Fig. 5. Illustration of detected area

\section{4) Circular list vector set}

We assume that two groups of camera sensors $\left(G_{1}, G_{2}\right)$ are randomly arranged in the expanded region $E R$ and a point $t$ is fall in the monitoring region $R$. Let $d(t)_{m}=\max \left\{d(t)_{1}, d(t)_{2}\right\}$ denotes the maximum detected area and $C L(t)$ represents the circular list vector set. We define the circular list vector set as the sequence of all sensors in the maximum area rotate in a counterclockwise or clockwise with point $t$ as the rotation axis. As illustrated in Fig. 6, the circular list vector sets of point $t$ is denoted as $C L(t)=\left\{t s_{1}, t s_{3}, t s_{6}, t s_{7}, t s_{5}, t s_{4}, t s_{2}\right\}$ 


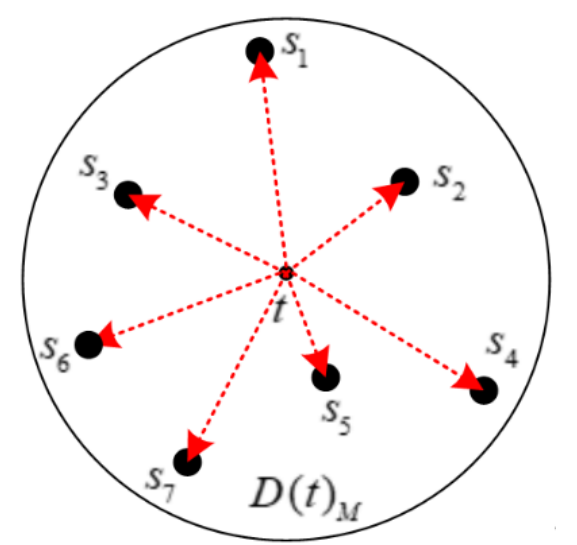

Fig. 6. Illustration of circular list vector set

Table 1. Description of Symbols

\begin{tabular}{|c|c|}
\hline Symbols & Description \\
\hline \hline$R$ & Monitoring Region \\
\hline$E R$ & Length of Monitoring Region \\
\hline$N_{i}$ & Scale of Group $i$ sensor \\
\hline$N=N_{1}+N_{2}$ & Scale of All Sensors \\
\hline$G_{i}$ & The Group $i$ sensor \\
\hline$r_{i}$ & Sensing Radius of $G_{i}$ \\
\hline$\varphi_{i}$ & Fov Angle of $G_{i}$ \\
\hline$C L(t)$ & Circular List Vector Set of Point $t$ \\
\hline$d(t)_{i}$ & Detected Area of $t$ with $G_{i}$ \\
\hline$d(t)_{M}$ & Maximum Detected Area \\
\hline$\theta$ & Effective Angle \\
\hline $\mathrm{P}$ & Rate of Full-View \\
\hline$\lambda_{i}$ & Sensor Density of $G_{i}$ \\
\hline$\lambda$ & Total Sensor Density \\
\hline
\end{tabular}

\section{Theorem 1. Judgement theorem of Full-view}

For a point $t$, let $t s_{v_{i}}$ and $t s_{v_{i+1}}$ denote any two adjacent vectors in $C L(t)$, respectively. Besides, we use $\mu\left(t s_{v_{i}}, t s_{v_{i+1}}\right)$ to represent the angle between $\mathbf{u s}_{v_{v_{i}}}$ and $t s_{v_{i+1}}$. If and if only $\mu$ satisfies the following condition $\mu\left(t s_{v_{i}}, t s_{v_{i+1}}\right) \leq 2 \theta, 1 \leq i \leq k, v_{k+1}=v_{1}$, we call that $t$ is full-view covered. 


\subsection{Symbols description}

To better describe the problem, the descriptions of symbols used in this paper are shown in Table 1.

\section{Sensor Density for Full-View in Heterogeneous Deployed CSNs}

This section mainly pay attention to deduce the sensor density prediction model in heterogeneous deployed CSNs with arbitrary full-view rate. Before the initial deployment, we can use the model to predict the density and parameter of sensor, so as to help engineers to deploy better CSNs.

Let $E_{1}$ and $E_{2}$ denote the event that point $t$ in the monitoring region $R$ is covered by sensor belongs to $G_{1}$ or $G_{2}$ located within the expanded region $E R$, respectively. It can be concluded that the probabilities of $E_{1}$ and $E_{2}$ are presented as follows.

$$
\begin{aligned}
& P\left(E_{1}\right)=\frac{\pi r_{1}^{2}}{|E R|} \times \frac{\varphi_{1}}{2 \pi} \\
& P\left(E_{2}\right)=\frac{\pi r_{2}^{2}}{|E R|} \times \frac{\varphi_{2}}{2 \pi}
\end{aligned}
$$

Where, $\frac{\pi r_{i}^{2}}{|E R|}, i=1,2$ denotes the probability of sensor belongs to $G_{i}$ fall into the detected area $d(t)_{i}$ corresponding to point $t ; \varphi_{i} / 2 \pi, i=1,2$ denotes the probability of sensing region of sensor belongs to $G_{i}$ towards $t$.

Let $B_{i}^{k_{i}}, i=1,2$ denotes the event in which the point $t$ is just covered by $k_{i}$ sensors belong to $G_{i}$ scattered in the expanded region $E R$, it can be seen that the event $B_{i}^{k_{i}}$ follows Bernoulli distribution denoted as $k_{i} \sim B\left(N_{i}, P\left(E_{i}\right)\right)$, the probabilities of event $\left(B_{i}^{k_{i}}, i=1,2\right.$ ) are expressed as the formulas.

$$
P\left(B_{i}^{k_{i}}\right)=\left(\begin{array}{l}
N_{i} \\
k_{i}
\end{array}\right)\left(P\left(E_{i}\right)\right)^{k_{i}}\left(1-P\left(E_{i}\right)\right)^{N_{i}-k_{i}}
$$

Lemma 1. It is supposed that massive sensors belong to $G_{1}$ and are scattered in the expanded region $E R$. In general, It can be concluded that $P\left(B_{i}^{k_{i}}\right)$ approximately follows Poisson distribution, and its strength is $\lambda_{i}\left|d(t)_{i}\right| q_{i}$. The Possion distribution can be expressed as $\lim _{N_{i} \rightarrow \infty} B_{i}^{k_{i}} \cong \mathrm{P}\left(k_{i}, \lambda_{i}\left|d(t)_{i}\right| q_{i}\right)$. Here, $\lambda_{i}=\frac{N_{i}}{|E R|}$ represents the density of sensors belong to $G_{i} ;\left|d(t)_{i}\right|=\pi r_{i}^{2}$ denotes the area of detected area of point $t$ corresponding to $G_{i}$ sensor; $q_{i}=\frac{\varphi_{i}}{2 \pi}$ presents the probability of sensing region of sensor belong to $G_{i}$ towards $t$. 
Proof:

$$
\begin{aligned}
\lim _{N_{i} \rightarrow \infty} & P\left(B_{i}^{k_{i}}\right) \\
& =\lim _{N_{i} \rightarrow \infty}\left(\begin{array}{l}
N_{i} \\
k_{i}
\end{array}\right)\left(P\left(E_{i}\right)\right)^{k_{i}}\left(1-P\left(E_{i}\right)\right)^{N_{i}-k_{i}} \\
& =\lim _{N_{i} \rightarrow \infty} \frac{N_{i} !}{\left(N_{i}-k_{i}\right) ! k_{i} !}\left(\frac{\pi r_{i}^{2}}{|E R|} \frac{\varphi_{i}}{2 \pi}\right)^{k_{i}}\left(1-\frac{\pi r_{i}^{2}}{|E R|} \frac{\varphi_{i}}{2 \pi}\right)^{N_{i}-k_{i}} \\
& =\lim _{N_{i} \rightarrow \infty} \frac{N_{i} !}{\left(N_{i}-k_{i}\right) ! N_{i}^{k_{i}}}\left(\frac{\lambda_{i}\left|d(t)_{i}\right| q_{i}}{k_{i} !}\right)^{k_{i}} \\
& \times\left(1-\frac{\lambda_{i}\left|d(t)_{i}\right| q_{i}}{N_{i}}\right)^{N_{i}}\left(1-\frac{\lambda_{i}\left|d(t)_{i}\right| q_{i}}{N_{i}}\right)^{-k_{i}} \\
& =e^{-\lambda_{i}\left|d(t)_{i}\right| q_{i}} \frac{\left(\lambda_{i}\left|d(t)_{i}\right| q_{i}\right)^{k_{i}}}{k_{i} !} \\
& =\mathrm{P}\left(k_{i}, \lambda_{i}\left|d(t)_{i}\right| q_{i}\right)
\end{aligned}
$$

Let $C^{k}$ represents the event in which point $t$ is just covered by $k=k_{1}+k_{2}$ sensors belong to $G_{1}$ and $G_{2}$. Based on the Equation (3) and the knowledge of probability, we derive the formula: $P\left(C^{k}\right)=\sum_{k} P\left(B_{1}^{k_{1}}\right) P\left(B_{2}^{k_{2}}\right)$.

Lemma 2. It is supposed that $\mathrm{P}(k, h)=e^{-h} \frac{(h)^{k}}{k !}$ in which $k=k_{1}+k_{2}>0$. Thus, $\sum_{k} \mathrm{P}\left(k_{1}, h_{1}\right) \mathrm{P}\left(k_{2}, h_{2}\right)=\mathrm{P}\left(k, h_{1}+h_{2}\right)$ for any positive integer $k_{1}$ and $k_{2}$.

Proof:

Taylor expands $e^{h x}$, thus

$$
\begin{aligned}
& e^{h x}=\sum_{j=0}^{\infty} \frac{h^{j}}{j !} x^{j}=\sum_{j=0}^{\infty} e^{h}\left(e^{-h} \frac{h^{j}}{j !}\right) x^{j} \\
& =\sum_{j=0}^{\infty} e^{h} \mathrm{P}(j, h) x^{j}
\end{aligned}
$$

Given $\prod_{i=1}^{2} e^{h_{i} x}=e^{\left(h_{1}+h_{2}\right) x}$, thus, we get that

$$
e^{\left(h_{1}+h_{2}\right) x}=\prod_{i}^{2} \sum_{j=0}^{\infty} e^{h_{i}} \mathrm{P}\left(j, h_{i}\right) x^{j}=\sum_{j=0}^{\infty} \frac{\left(h_{1}+h_{2}\right)^{j}}{j !} x^{j} .
$$

Then, from the above formula, we know that 


$$
\sum_{k} e^{\left(h_{1}+h_{2}\right)} \mathrm{P}\left(k_{1}, h_{1}\right) \mathrm{P}\left(k_{2}, h_{2}\right)=\frac{\left(h_{1}+h_{2}\right)^{k}}{k !}
$$

Finally, $\sum_{k} \mathrm{P}\left(k_{1}, h_{1}\right) \mathrm{P}\left(k_{2}, h_{2}\right)=\mathrm{P}\left(k, h_{1}+h_{2}\right)$.

Lemma 3. Based on the Lemma 1 and Lemma 2, we obtain the following formula.

$$
P\left(C^{k}\right) \cong \mathrm{P}\left(k, \sum_{i}^{2} \lambda_{i}\left|d(t)_{i}\right| q_{i}\right)
$$

Theorem 2. Let $F_{t}$ denotes the event in which point $t$ is full-view covered by sensors belong to $G_{1}$ and $G_{2}$. According to the Theorem 1-Lemma 3, It can be known that the probability of $F_{t}$ is represented as follow.

$$
\mathrm{P}=P\left(F_{t}\right)=\sum_{k=\lceil\pi / \theta\rceil}^{N} P\left(C^{k}\right) P\left(U^{k}\right)
$$

Where, $\lceil\pi / \theta\rceil$ denotes the least number of sensors in $d(t)_{m}$ corresponding to target $t$; $U^{k}$ indicates the event in which circumference of a circle of unit length is just covered by $k$ uniformly distributed arcs of length $\theta / \pi$. From the literature [5], we can know that expression of the probability of $U^{k}$ is as follow.

$$
p\left(U^{k}\right)=\sum_{j=0}^{\lceil\pi / \theta\rceil}\left(\begin{array}{l}
k \\
j
\end{array}\right)(-1)^{j}\left(1-\frac{j \theta}{\pi}\right)^{k-1} .
$$

From this formula, given a full-view rate $\mathrm{P}$ and a sensor scale's heterogeneous proportion $\rho=\frac{N_{1}}{N_{2}}$, we can calculate the number of $G_{1}$ and $G_{2}$ camera sensor needed to be deployed in expanded region $E R$ respectively. Thus, the total sensor density and the sensor density of $G_{1}$ and $G_{2}$ in CSNs respectively denoted as $\lambda, \lambda_{1}$ and $\lambda_{2}$, can be computed by the following formulas.

$$
\begin{aligned}
& \lambda=\frac{(1+\rho) N_{2}}{|E R|} \\
& \lambda_{1}=\frac{N_{1}}{|E R|} \\
& \lambda_{2}=\frac{N_{2}}{|E R|}
\end{aligned}
$$


Example 1. Assume that the heterogeneous proportion of sensor scale $\rho=\frac{N_{1}}{N_{2}}$ is equal to 1 , and massive sensros belong to $G_{1}$ and $G_{2}$ are stochastically scattered in the expanded region $E R$ to monitor the targets (number is 20) in monitoring region $R$ with 0.99 full-view rate. For simplicity, let $L=100 * 100, \theta=60^{\circ}, r_{1}=10, \gamma_{2}=8, \varphi_{1}=60^{\circ}, \varphi_{2}=48^{\circ}$, thus, we get the expanded region $E R=118 * 118$. Based on the Theorem 2 and Equations (7)(8)(9), it can be estimated that $\lambda_{1}=\lambda_{2}=0.15, \lambda=0.3$.

\section{Simulation and Performance Analysis}

In this section, Matlab 2018a is adapted to set up simulation scenario to validate the proposed model. We suppose that massive sensors belong to $G_{1}$ and $G_{2}$ are independently scattered in the expanded region to monitor the targets in monitoring region. A series of simulation experiments (also called experimental results) are carried out to verify the performance of the model theoretical results and scenario simulation results. Aiming to get more precise experimental results of simulation scenario, each group of simulation experiments is randomly repeated $m=100$ times, the simulation average result (SAR) is presented as follow.

$$
S A R=\frac{L_{+}+?_{2}++{ }_{m}}{m}
$$

Where, $\mathrm{i}_{i}, 1 \leq i \leq m$ is the $i^{\text {th }}$ simulation full-view rate of each scenario.

Table 2. Experimental parameters

\begin{tabular}{|l|c|}
\hline \multicolumn{1}{|c|}{ Parameter } & Variation \\
\hline Length of monitoring region, $L$ & $100 \mathrm{~m}$ \\
\hline Number of targets & 20 \\
\hline Sensing radius proportion, $r_{1} / r_{2}$ & 0.6 or 0.8 \\
\hline Fov angle proportion, $\varphi_{1} / \varphi_{2}$ & 0.6 or 0.8 \\
\hline Effective angle, $\theta$ & $45^{\circ}$ or $60^{\circ}$ \\
\hline
\end{tabular}

In our simulations, we analyze the simulation results and theoretical results from three aspects, including sensor density, sensing radius, and field-of-view (Fov) angle. In order to make these experiments simpler, we set the length of monitoring region $R$ is 100 , and assume that 20 points is located within the monitoring region $R$. The main parameters of experiment are shown in Table 2. 


\subsection{Impact of the sensor density}

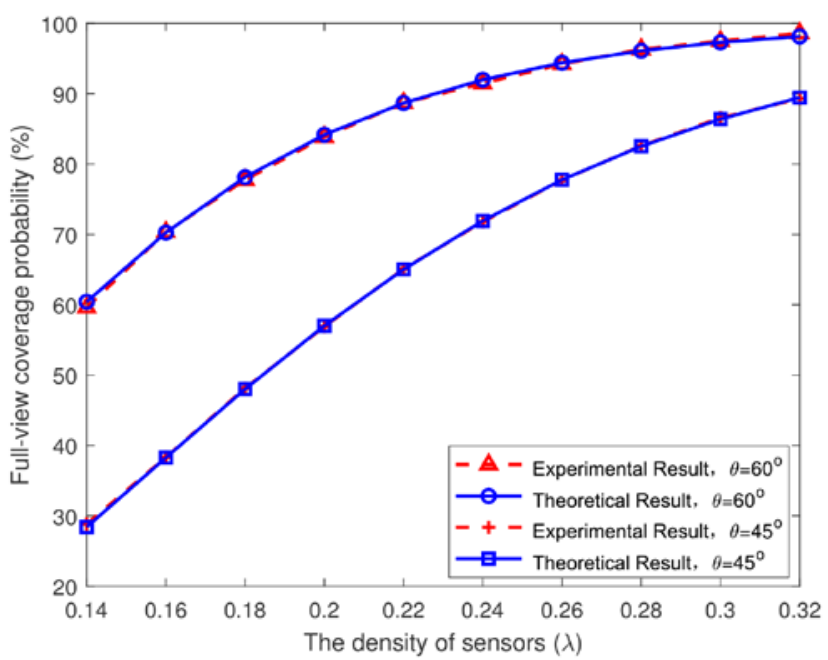

(a)

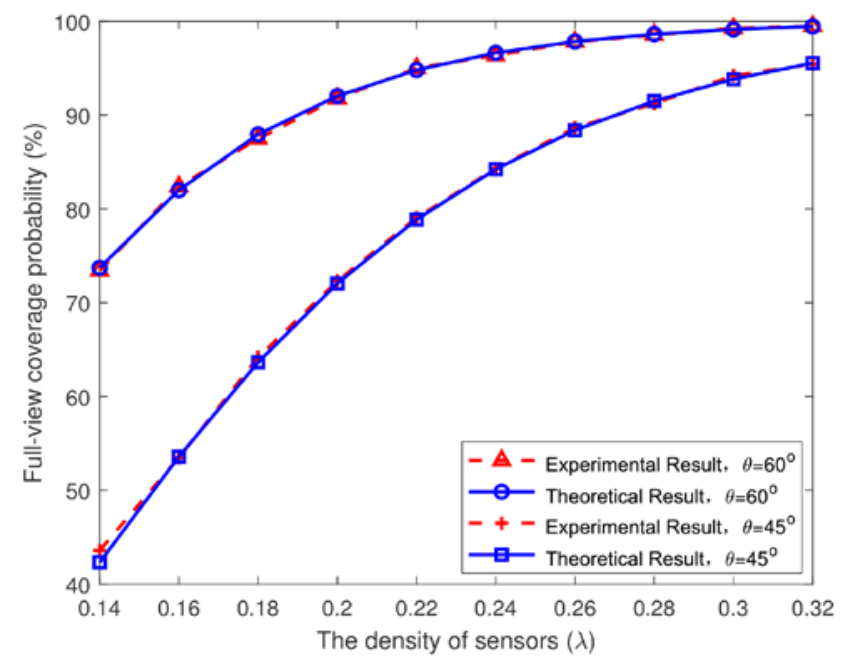

(b)

Fig. 7. Analysis of experimental and theoretical values under different sensor density $\lambda$, (a)

$$
r_{1} / r_{2}=\varphi_{1} / \varphi_{2}=0.6 \text {, (b) } r_{1} / r_{2}=\varphi_{1} / \varphi_{2}=0.8
$$

In this experiment, we analyze the effect of sensor density on full-view rate. The parameters of this experiment are set to $r_{1}=10, \varphi_{1}=60^{\circ}$ and $N_{1}=N_{2}$.

Fig. 7(a) shows the simulation results and theoretical results of full-view rate under different effective angle $\theta=45^{\circ}$ or $\theta=60^{\circ}$ and sensor density. We can calculate $r_{2}$ and $r_{2}$ according to the heterogeneous proportions $r_{1} / r_{2}=\varphi_{1} / \varphi_{2}=0.6$. In both cases of different effective angles, from the trend of Fig. 7(a), we can know that with the increase of sensor density $\lambda$, the full-view rate increases, and the errors between theoretical and simulation 
values are very small. For example, given the full-view rate 0.99 and the effective angle $\theta=60^{\circ}$, according to the propose model, we calculate the sensor density needed to achieve 0.99 is approximately 0.32 . Further more, at the case with the same sensor density, the fullview rate increases as the effective angle due to the effective angle is larger, the less sensors are needed to achieve full-view coverage.

Fig. 7(b) shows the trend when the heterogeneous proportions $r_{1} / r_{2}=\varphi_{1} / \varphi_{2}=0.8$, the analysis conclusion are similar to those of Fig. 7(a).

\subsection{Impact of the sensing radius}

In this experiment, we analyze the effect of sensing radius of $G_{1}$ on full-view rate according to different $r_{1} / r_{2}=\varphi_{1} / \varphi_{2}$ and $N_{1}$. We also assume that $N_{1}=N_{2}$.

The relative parameters of this group of experiments are set as follows: $r_{1} / r_{2}=\varphi_{1} / \varphi_{2}=0.6$ and $N_{1}=N_{2}=800$ or $N_{1}=N_{2}=1400$. As illustrated in Fig. 8(a) -

Fig. 8(b), it is concluded that no matter what the effective angle is $\theta=45^{\circ}$ or $\theta=60^{\circ}$, the full-view rate increments with the increment of sensing radius. Meanwhile, the fit degree between theoretical and experiment values is very good. It means that the error between them is approximately equal to zero. When the sensing radius $r_{1}$ of $G_{1}$ is large enough(such as $r_{1}=14$ ) and the effective angle $\theta=60^{\circ}$, the full-view rate is approximately 1 .

In order to further verify fit degree between theoretical and experiment results, we deploy $N_{1}=N_{2}=800$ or $N_{1}=N_{2}=1400$ groups ( $G_{1}$ and $G_{2}$ ) camera sensors with $r_{1} / r_{2}=\varphi_{1} / \varphi_{2}=0.8$ in the expanded region $E R$. From Fig. 8(c) - Fig. 8(d), we can know that the error between theoretical and experiment values is in a small range.

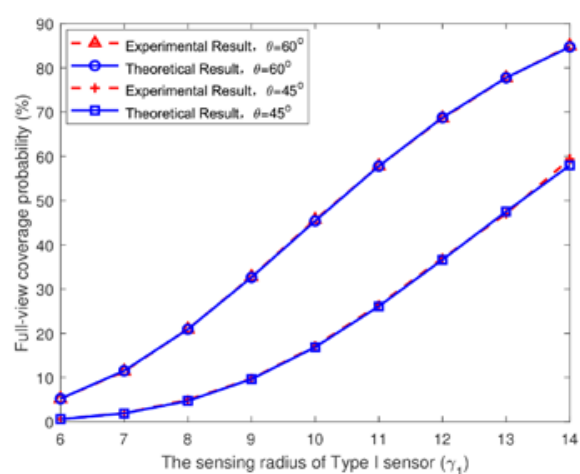

(a)

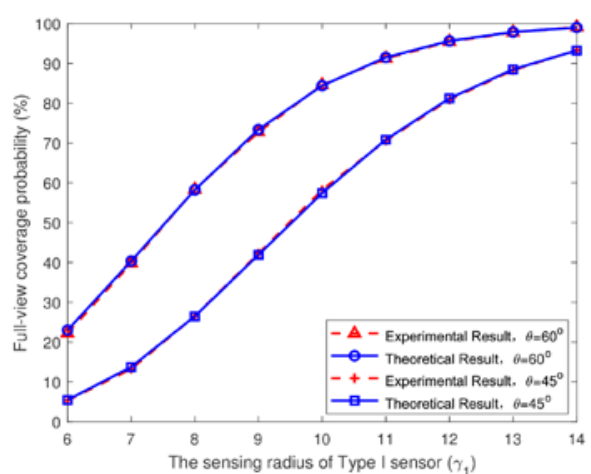

(b) 


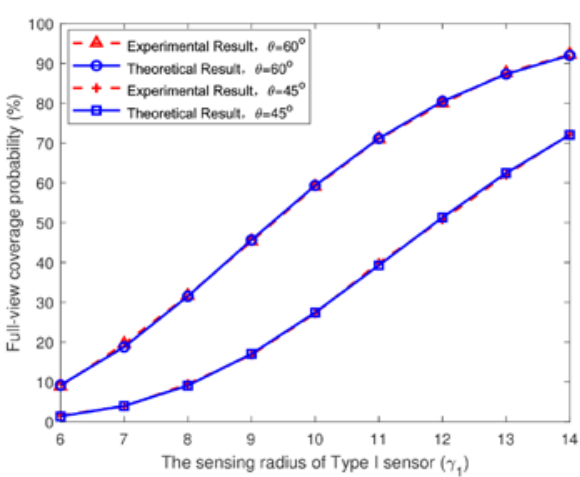

(c)

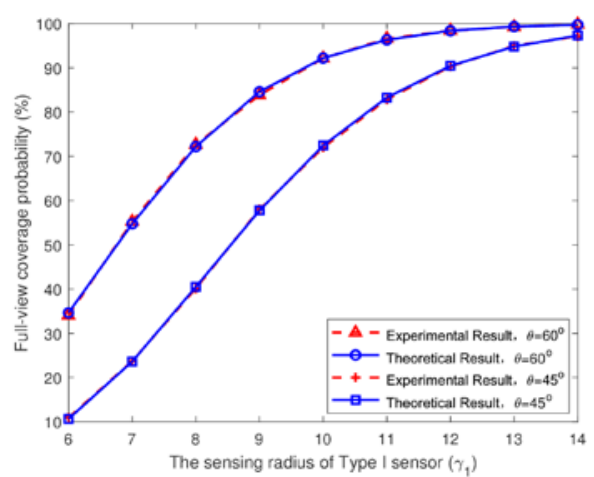

(d)

Fig. 8. Analysis of experimental and theoretical values under sensing radius $r_{1}$ of $G_{1}$ sensor, (a)

$$
\begin{gathered}
r_{1} / r_{2}=\varphi_{1} / \varphi_{2}=0.6, N_{1}=800, \text { (b) } r_{1} / r_{2}=\varphi_{1} / \varphi_{2}=0.6, N_{1}=1400, \text { (c) } \\
r_{1} / r_{2}=\varphi_{1} / \varphi_{2}=0.8, N_{1}=800, \text { (d) } r_{1} / r_{2}=\varphi_{1} / \varphi_{2}=0.8, N_{1}=1400 .
\end{gathered}
$$

\subsection{Impact of the Fov angle}

In this experiment, we analyze the effect of Fov angle on full-view rate. The same number of sensors belong to $G_{1}$ and $G_{2}$ are arranged in the expanded region $E R$. Different $N_{1}$ and $r_{1} / r_{2}=\varphi_{1} / \varphi_{2}$ are selected to validate accuracy of proposed model.

In the first experiment (Results shown in Fig. 9(a) -Fig. 9(b)), we set the parameters of this experiment as $r_{1} / r_{2}=\varphi_{1} / \varphi_{2}=0.6$, and the number of $G_{1}$ is $N_{1}=800$ or $N_{1}=1400$. For different effective angle $\theta=45^{\circ}$ or $\theta=60^{\circ}$, the results show that the full-view rate increments as the Fov angle, and the error between theoretical and experimental values is controlled in a small range.

We also deploy $N_{1}=N_{2}=800$ or $N_{1}=N_{2}=1400$ groups $\left(G_{1}\right.$ and $\left.G_{2}\right)$ camera sensors with $r_{1} / r_{2}=\varphi_{1} / \varphi_{2}=0.8$ in expanded region ER. From Fig. 9(c) - Fig. 9(d), we can know that the fit degree between theoretical and experimental values is good no matter what the effective angle $\theta=45^{\circ}$ or $\theta=60^{\circ}$.

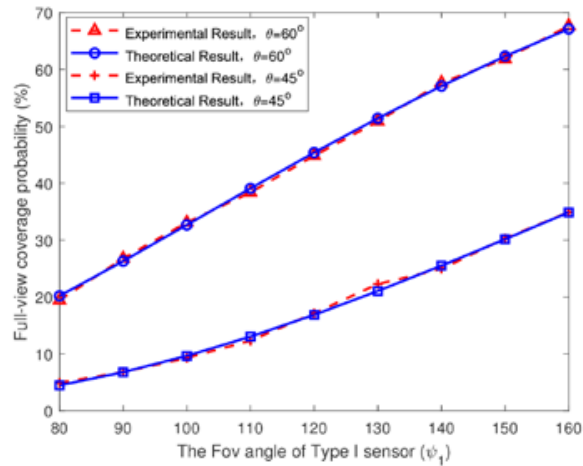

(a)

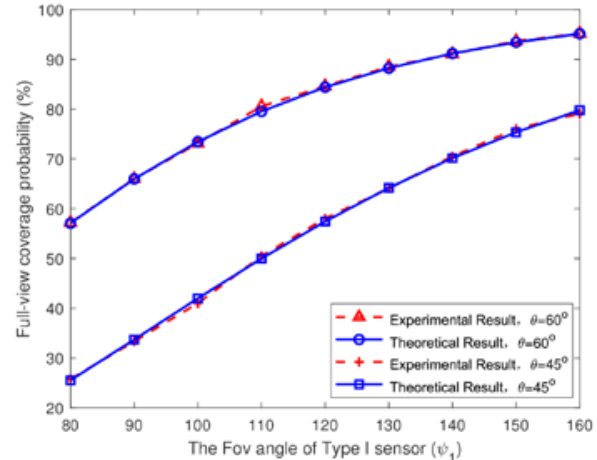

(b) 


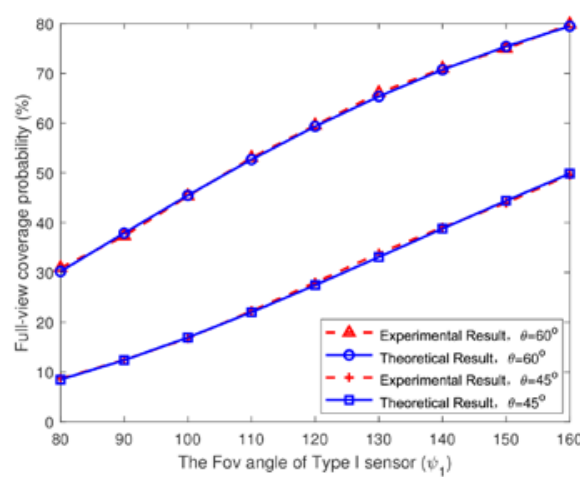

(c)

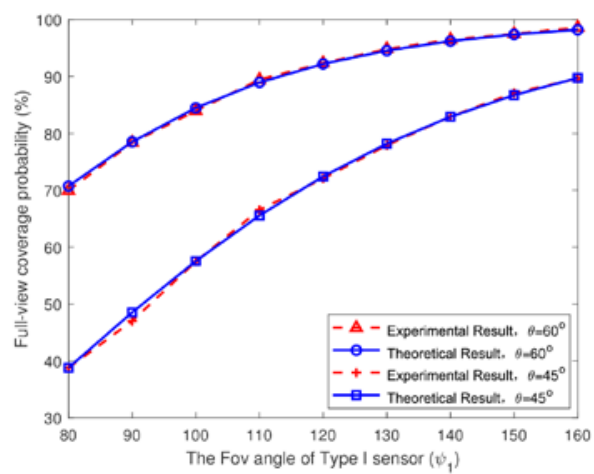

(d)

Fig. 9. Analysis of experimental and theoretical values under Fov angle $\varphi_{1}$ of $G_{1}$ sensor,

$$
\begin{aligned}
\text { (a) } r_{1} / r_{2} & =\varphi_{1} / \varphi_{2}=0.6, N_{1}=800, \text { (b) } r_{1} / r_{2}=\varphi_{1} / \varphi_{2}=0.6, N_{1}=1400, \text { (c) } \\
r_{1} / r_{2} & =\varphi_{1} / \varphi_{2}=0.8, N_{1}=800, \text { (d) } r_{1} / r_{2}=\varphi_{1} / \varphi_{2}=0.8, N_{1}=1400 .
\end{aligned}
$$

\section{Conclusion}

In this paper, we study the sensor density prediction problem in heterogeneous deployed CSNs with arbitrary full-view rate. Aiming to reduce the influence of boundary effect, we proposed the concepts of expanded monitoring region and maximum detected area. Furthermore, we derive the sensor density model which expresses the mathematical relationship among sensor density, sensing radius, Fov angle and effective angle. Compared with experimental and theoretical values, we demonstrate that the error between them is controlled in a small range. Our research results have a certain guiding significance for the analysis and design of CSNs. However, in the future work, there are some problems worthy of attention, such as the enhancement problem of full-view in mobile heterogenous deployed CSNs.

\section{References}

[1] B. Cheng et al., "Multiple Region of Interest Coverage in Camera Sensor Networks for TeleIntensive Care Units,” IEEE Transactions on Industrial Informatics, vol. 126, no. 6, pp. 2331-2341, 2016. Article (CrossRef Link)

[2] N. Kumar, J.H. Lee and J.J.P.C Rodrigues, "Intelligent Mobile Video Surveillance System as a Bayesian Coalition Game in Vehicular Sensor Networks: Learning Automata Approach,” IEEE Transactions on Intelligent Transportation Systems, vol. 16, no. 3, pp. 1148-1161, 2015.

Article (CrossRef Link)

[3] K.R. Konda, J. Conci and F.D. Natale, "Global Coverage Maximization in PTZ-Camera Networks Based on Visual Quality Assessment,” IEEE Sensors Journal, vol. 16, no. 16, pp. 6317-6332, 2016. Article (CrossRef Link)

[4] H.D. Ma and D. Tao, "Multimedia sensor network and its research progresses," Journal of Software (in chinese journal), vol. 17, no. 9, pp. 2013-2028, 2006. Article (CrossRef Link)

[5] Y. Wang and G.H. Cao, “On full-view coverage in camera sensor networks," in Proc. of INFOCOM, pp. 1781-1989, 2011. Article (CrossRef Link) 
[6] Y. Wang and G.H. Cao, “Achieving Full-View Coverage in Camera Sensor Networks,” ACM Transactions on Sensor Networks, vol. 10, no. 1, pp. 1-31, 2013. Article (CrossRef Link)

[7] Y.T. Hu, X.B. Wang and X.Y. Gan, "Critical sensing range for mobile heterogeneous camera sensor networks,” in Proc. of INFOCOM, pp. 970-978, 2014. Article (CrossRef Link)

[8] X.Y. Gan et al., "Unraveling Impact of Critical Sensing Range on Mobile Camera Sensor Networks,” IEEE Transactions on Mobile Computing, vol. 19, no. 4, pp. 982-996, 2020. Article (CrossRef Link)

[9] Z.M. Yu et al., "Local face-view barrier coverage in camera sensor networks," in Proc. of INFOCOM, pp. 684-692, 2015. Article (CrossRef Link)

[10] Y. Wang and G.H. Cao, "Barrier coverage in camera sensor networks," in Proc. of MOBIHOC, pp. 1-12, 2011.

[11] X. Liu, "Full-view barrier coverage in mobile camera sensor networks," Wireless, Networks, vol. 25, pp. 4773-4784, 2019. Article (CrossRef Link)

[12] R. Yang et al., "Distributed Algorithm for Full-View Barrier Coverage with Rotatable Camera Sensors,” in Proc. of GLOBECOM, pp. 1-6, 2014. Article (CrossRef Link)

[13] C. Gan et al., "Self-orienting the cameras for maximizing the view-coverage ratio in camera sensor networks,” Pervasive and Mobile Computing, vol. 17, no. 17, pp. 102-121, 2015. Article (CrossRef Link)

[14] S.B. He et al., "Full-View Area Coverage in Camera Sensor Networks: Dimension Reduction and Near-Optimal Solutions,” IEEE Transactions on Vehicular Technology, vol. 65, no. 9, pp. 74487461, 2016. Article (CrossRef Link)

[15] Q. Zhang, S.B. He and J.M. Chen, "Toward Optimal Orientation Scheduling for Full-View Coverage in Camera Sensor Networks,” in Proc. of GLOBECOM, pp. 1-6, 2016. Article (CrossRef Link)

[16] J.M. Chen et al., "Orientation Optimization for Full-View Coverage Using Rotatable Camera Sensors,” IEEE Internet of Things Journal, vol. 6, no. 6, pp. 10508-10518, 2019. Article (CrossRef Link)

[17] Z.M. Liu et al., "k -Coverage Estimation Problem in Heterogeneous Camera Sensor Networks With Boundary Deployment,” IEEE Access, vol. 6, pp. 2825-2833, 2017. Article (CrossRef Link)

[18] P.K. Khan et al., “Social Network chatting apps network traffic optimization,” Indian Journal of Science and Technology, vol. 11, pp. 1-6, 2017. Article (CrossRef Link).

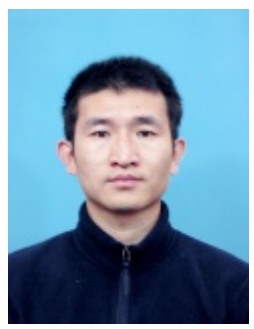

Zhimin Liu was born in Chenzhou, China. He received the B.E. degree from Northeastern University and the M.E. degree from South China Agricultural University in 2006 and 2010, and Ph.D. degree from Central South University 2017, respectively. Now he is a lecturer of School of Mathematics and Computational Science at Hunan First Normal University. His current interests include wireless camera sensor network, sensor cloud, crowd sensing, etc.

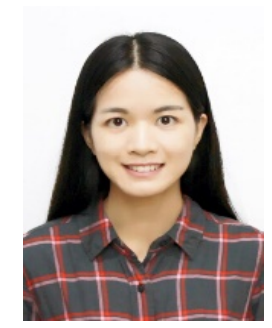

Guiyan Jiang was born in Shaoyang, China. She received the B.E. degree from South China Agricultural University in 2011, and the M.E. degree from Hunan University in 2019. Now, She is a doctoral student, in school of economics university, Wuhan University of Technology, Wuhan, China. Her research interests include Internet economy and mult-agent si mulation. 